\title{
Characterization of Microcystis
} (Cyanobacteria) Genotypes Based on the Internal Transcribed Spacer Region of rRNA by Next-Generation Sequencing

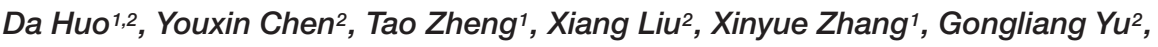 \\ Zhiyi Qiao ${ }^{\text {* }}$ and Renhui $\mathrm{Li}^{2 *}$
}

${ }^{1}$ National Demonstration Center for Experimental Aqua-ecology and Aquaculture Education, Department of Fisheries Sciences, Tianjin Agricultural University, Tianjin, China, ${ }^{2}$ Key Laboratory of Algal Biology, Institute of Hydrobiology, Chinese Academy of Sciences, Wuhan, China

OPEN ACCESS

Edited by:

Sandra M. F. O. Azevedo, Institute of Biophysics Carlos Chagas

Filho (IBCCF), Brazil

Reviewed by:

Rachel Susan Poretsky, University of Illinois at Chicago,

United States

Fabiano Thompson, Instituto Alberto Luiz Coimbra de Pós-Graduação e Pesquisa de Engenharia (COPPE), Brazil

*Correspondence: Zhiyi Qiao qiaozhiyi7070@163.com

Renhui Li reli@inb.ac.cn

Specialty section:

This article was submitted to

Aquatic Microbiology,

a section of the journal

Frontiers in Microbiology

Received: 10 November 2017 Accepted: 25 April 2018 Published: 15 May 2018

Citation: Huo D, Chen Y, Zheng T, Liu X, Zhang $X, Y u G$, Qiao $Z$ and $L i ~ R$ (2018) Characterization of Microcystis (Cyanobacteria) Genotypes Based on the Internal Transcribed Spacer Region of rRNA by Next-Generation Sequencing. Front. Microbiol. 9:971. doi: 10.3389/fmicb.2018.00971
Microcystis is one of the most common and dominant bloom-forming cyanobacteria in freshwater worldwide. The method for genotype detection based on traditional molecular cloning is expensive and time consuming and generates a limited number of sequences. In this study, a high-throughput sequencing (HTS) method was developed to detect the internal transcribed spacer (ITS) regions between 16S and 23S rRNA region of Microcystis populations along a typical water system in Yuqiao Reservoir-Haihe River in Tianjin, northern China. A total of 629,341 reads were obtained and clustered into 2005 operational taxonomic units (OTUs). Analysis of alpha diversity indices showed that the Haihe River is more diverse than Yuqiao Reservoir. In general, the two water areas exhibit a clear differentiation pattern in OTU abundance, sharing genotypes from a small part of Yuqiao Reservoir with those in the Haihe River. Phylogenetic analysis further indicated the possible flexible evolution of Microcystis genotypes occurring in the research areas. This study provides the first exhaustive description of HTS method for detection of ITS region to evaluate Microcystis intra-species diversity and relationship.

Keywords: cyanobacteria, Yuqiao reservoir, Haihe River, high throughput sequencing, ITS region

\section{INTRODUCTION}

Cyanobacteria are prokaryotes regarded as the oldest oxygenic photosynthetic organisms on earth (Hess, 2011; Wittmann and Liao, 2016). With a broad adaption in most living habitats, especially, prominent standing capacity for extreme environment (Cristiana et al., 2013; Klanchui et al., 2017), cyanobacteria are widely distributed worldwide (Whitton, 2012; Visser et al., 2016). They are abundant components of aquatic ecosystems, particularly in eutrophicated waters, and may form blooms in water bodies (Chislock et al., 2013). Cyanobacterial blooms have become a serious global issue in fresh water lakes, rivers, and reservoirs. Bloom-forming cyanobacteria can form scums and odorous compounds and produce toxic metabolites, such as microcystins (MCs) (Codd et al., 2005; Paerl et al., 2015). A combination of multiple biological, physicochemical, and climatic factors causes the development of harmful cyanobacterial blooms. Eutrophication of water bodies is the main factor promoting development of cyanobacterial bloom in the past decades (Bonilla et al., 2012; O'neil et al., 2012). However, global climate change and the rise of carbon 
dioxide concentration have been regarded as important factors that trigger the extensive development of cyanobacterial blooms as well (Paerl and Huisman, 2008; Verspagen et al., 2014). MCs are hepatotoxins and tumor promoters that are mainly produced by Microcystis, a unicellular colony-forming cyanobacterial group. Microcystis-dominated blooms have largely occurred worldwide, Therefore, numerous studies have been focused on Microcystis-dominated blooms and their harmful effects (Li et al., 2012; Srivastava et al., 2012).

The genetic diversity, composition, and spatiotemporal dynamics of cyanobacteria populations have been widely studied to further understand their proliferation. Sequencing of a specific gene marker provides a way to track the dynamic variation in cyanobacterial communities (Stucken et al., 2009). Because of its wide distribution and harmful influence, the genetic characteristics of Microcystis have been studied extensively. (Nübel et al., 1997; Zhang et al., 2009; Tan et al., 2010; Xu et al., 2010). Microcystis was shown to be highly diverse at the intra-species level not only among geographically distant ecosystems but also during bloom proliferation in localized freshwater environments (Kataoka et al., 2013). The internal transcribed spacer (ITS) region of 16S-23S rRNA is an effective molecular marker for characterization of Microcystis intra-species diversity (Yoshida et al., 2008; Moreira et al., 2016), and several studies used ITS region as gene marker to examine the cyanobacterial community structures (Otten et al., 2015). Compared with the Oligotypes of 16S rRNA gene used in Lake Erie (Berry et al., 2017), ITS provide a higher genetic resolution of Microcystis strains (Briand et al., 2009; Xu et al., 2010; Lemaire et al., 2012; Liu et al., 2016). However, Zhu et al. (2012) indicated that no significantly dominant genotypes existed in Xinghu Pond, a small eutrophic pond in central China, partially consistent with the finding by Pobel et al. (2012) who found no spatial ITS heterogeneity of each sampling date in a french shallow lake. These conflicting results implied that complex circumstances and the multiple factors may drive the genetic composition of Microcystis populations in water system. Recently, researchers sequenced cyanobacterial ITS region by 454 pyrosequencing, and found eight Microcystis genotypes in these sequences. Even using pyrosequencing method, the sequencing depth is still not enough to uncover the high diversity of Microcystis, so that several PCR sequences could not be found in these eight OTUs (Otten et al., 2017). The primers specific for Microcystis is needed in these ecological studies.

The development of whole genome based taxonomic system should be the ideal goal for studying cyanobacterial systematics and diversity (Thompson et al., 2013; Soo et al., 2014; Walter et al., 2017). For elucidating the diversity in a specific cyanobacterial group such as Microcystis, analyzing a single gene is still the most convenient way. By using molecular cloning method, Sabart et al. (2009) found that most Microcystis genotypes seem to be ubiquitous. Nevertheless, low efficiency of this traditional cloning method causes difficulty in identifying the comprehensive diversity of Microcystis. The limited numbers of clones may lead to an underestimated or biased evaluation of Microcystis genotypes. With the development of high-throughput sequencing (HTS) techniques, current methods enable researchers to achieve faster and less costly genomic characterizations of cyanobacterial strains and raise additional information about their associated microorganisms (Danillo et al., 2017). Several studies used deep targeted sequencing as a powerful tool to detect the microbial community structure and cyanobacterial composition during cyanobacterial bloom period (Chen et al., 2011; Bertos-Fortis et al., 2016; Parulekar et al., 2017), And by improving the resolution of gene marker sequencing (Ruegger et al., 2014), Next-generation sequencing targeting the 16S rRNA gene now allows the comprehensive investigation of bacterial community composition with coverage far beyond that of previous clone libraries (Mackey et al., 2017). The effectiveness or advantage of ITS region-targeted HTS in detecting of Microcystis intra-species diversity remains not clear.

Yuqiao Reservoir, the drinking water sources of Tianjin City, China, supports more than one million people's water usage. Yuqiao Reservoir is at the upper part of Haihe River flowing through the Tianjin city. The proliferation of Microcystis threats both Yuqiao Reservoir and downstream Haihe River (Huo et al., 2018). However, the evaluation and high-frequency monitoring of cyanobacterial blooms in Yuqiao Reservoir-Haihe River system remain uncharted. Even two water areas are connecting each other, they are shown to have different levels of eutrophication. The investigation on the diversity of Microcystis between these two waters will help to understand the selective mechanism on Microcystis under different eutrophication levels. This study aims to characterize Microcystis genotypes based on the ITS region of rRNA through next-generation sequencing. To comprehensively discover the potential diversity of Microcystis ITS region, we designed new primers based on reported sequences that are currently available in public databases. We collected water samples from cyanobacterial blooms along the Yuqiao-Haihe water system in Tianjin, northern China. Deep targeted sequencing was performed on these Microcystis-based blooms. The study was expected to provide information on discussing the feasibility of HTS in studying Microcystis ITS region diversity.

\section{MATERIALS AND METHODS}

\section{Sampling Strategy}

Surface water samples from 15 sites in Yuqiao Reservoir and Haihe River were collected in September 2016. The locations of the sampling sites are shown in Figure 1. Dissolved oxygen, water temperatures (WT), salinity (SAL), and $\mathrm{pH}$ were measured using YSI multi-parameter detector (YSI, United States). Chlorophyll a, total nitrogen (TN), total phosphorus (TP), chemical oxygen demand, $\mathrm{NH}_{4}^{+}$, and $\mathrm{NO}_{2}^{-}$were analyzed according to standard methods (Jin and $\mathrm{Tu}, 1990)$. Water $(500 \mathrm{ml})$ was filtered through a $0.22 \mu \mathrm{m}$ filter (Millipore, United States) for HTS at each sampling sites. The filtrate was stored at $-80^{\circ} \mathrm{C}$ until further analysis.

\section{DNA Extraction and Library Construction}

DNA was extracted from each filtered membrane using the PowerWater DNA isolation kit following the manufacturer's 


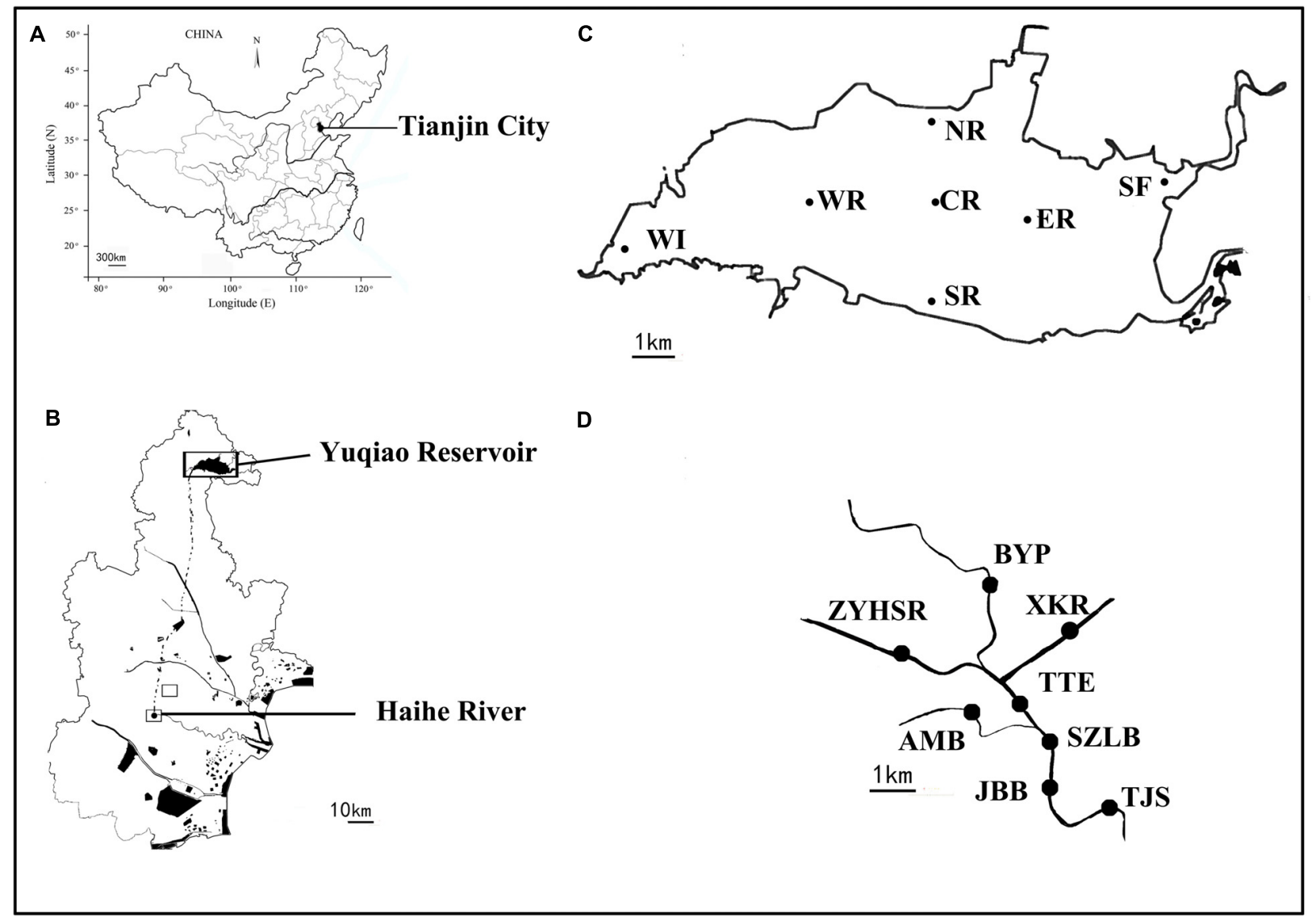

FIGURE 1 | Distribution of sampling sites between two water areas. Dotted line shows the underground connecting channel between two water areas. Maps of (A) China, (B) Tianjin city, (C) Yuqiao reservoir, and (D) Haihe River. Adapted from Huo et al. (2018).

manual (Mo Bio Laboratories, Inc., Carlsbad, CA, United States). DNA concentration was evaluated using Qubit 2.0 fluorometer (Life Technologies Japan Ltd., Tokyo, Japan). We designed a specific primer with a suitable length for amplification due to limited read length on Illumina Miseq ${ }^{\mathrm{TM}}$ platform. We designed the forward primer HTSITS_F as 5' -TACACGACGCTCTTCCGATCTCTAG(barcode)AAGGGAGACCTAAT TCRGGTA-3', and used the reverse primer ITS_R as $5^{\prime}$ TGGAGTTCCTTGGCACCCGAGAA- TTCCATAGCCTCTG TGTGCCTAGGTATCC-3' (Iteman et al., 2000). Both primers contain an Illumina adapter region for sequencing on the Illumina Miseq ${ }^{\mathrm{TM}}$ platform. A 6 bp barcode in the forward primer was used to distinguish each sample. And the length of PCR products was about $550 \mathrm{bp}$. We checked the sensitivity of primers by using different cyanobacterial species as template for amplification. The result of primer specificity test is shown in Supplementary Table S1.

\section{Processing of OTUs}

The quality filtering and processing of raw data were performed with cut adapt (Martin, 2011), PEAR (Zhang et al., 2014), and Prinseq (Schmieder and Edwards, 2011). We used Uchime to check and remove chimeras (Edgar et al., 2011). All samples were assigned using UPARSE method at 97\% cut-off (Edgar, 2013). A Venn diagram was constructed to reflect the common OTU and unique OTU numbers in all samples using $\mathrm{R}$ package Venn Diagram. To further examine the specificity of primers that we designed, we used Online BLAST (Lobo, 2012) search tool to assign the taxonomy of all OTUs with the NT database of NCBI. Sequences with a similarity of $>90 \%$ and coverage of $>90 \%$ were considered as identified or interpreted as unclassified if otherwise. A summary of taxonomic result is shown in Supplementary Table S2.

\section{Phylogenetic Analyses}

A phylogenetic tree was constructed using the top 20 abundantly represented sequences of OTUs in this study and 100 other reported representative sequences. Sequences were summarized by Song et al. (2015). All sequences were aligned with Bioedit (version 7.2.6.1) (Hall, 1999), and a neighbor-joining tree (Saitou and Nei, 1987) was constructed using MEGA (version 7.0) (Kumar et al., 2008). Evolutionary distances were computed using the Kimura 2-parameter method. Bootstrap trial was set to 1000 . The final tree figure was annotated using $\mathrm{R}$ package ggtree ( $\mathrm{Yu}$ et al., 2017). 
TABLE 1 | Detailed information of sequencing result and diversity indices.

\begin{tabular}{|c|c|c|c|c|c|c|c|}
\hline Sample & Seq num & OTU & Shannon & ACE & Chao1 & Coverage & Simpson \\
\hline HH_AMB & 35082 & 508 & 2.801632 & 793.818389 & 771.129412 & 0.993957 & 0.155512 \\
\hline HH_BYP & 28440 & 540 & 2.655934 & 1105.595904 & 849.141304 & 0.991596 & 0.182228 \\
\hline HH_JBB & 38661 & 533 & 2.78349 & 1073.375118 & 816.453608 & 0.993922 & 0.156036 \\
\hline HH_SZLB & 30003 & 506 & 2.376125 & 803.330239 & 709.584071 & 0.992834 & 0.238113 \\
\hline HH_TJS & 25791 & 588 & 2.909642 & 891.559234 & 854.342857 & 0.990811 & 0.169032 \\
\hline HH_TTE & 33573 & 371 & 2.082338 & 664.749925 & 520.013699 & 0.995592 & 0.268606 \\
\hline $\mathrm{HH} \_\mathrm{XKR}$ & 38539 & 271 & 1.284971 & 552.733938 & 398.258621 & 0.996834 & 0.570362 \\
\hline HH_ZYHSR & 39169 & 664 & 3.249824 & 1198.519414 & 983.918919 & 0.993183 & 0.105657 \\
\hline$Y Q_{-} C R$ & 34386 & 328 & 2.169507 & 824.480111 & 562.491228 & 0.995231 & 0.190352 \\
\hline YQ_ER & 40823 & 309 & 1.968443 & 686.566003 & 488.40678 & 0.996424 & 0.225406 \\
\hline YQ_NR & 33220 & 288 & 1.889695 & 809.701961 & 484.981132 & 0.995635 & 0.229471 \\
\hline YQ_SF & 39158 & 388 & 1.774099 & 881.712147 & 589.658228 & 0.995429 & 0.35491 \\
\hline YQ_SR & 40429 & 418 & 2.253835 & 833.221758 & 641.441558 & 0.995399 & 0.22677 \\
\hline YQ_WI & 33344 & 245 & 1.920372 & 567.094033 & 406.333333 & 0.996371 & 0.225656 \\
\hline YQ_WR & 35207 & 305 & 1.510009 & 670.809446 & 456.681818 & 0.995967 & 0.391206 \\
\hline
\end{tabular}

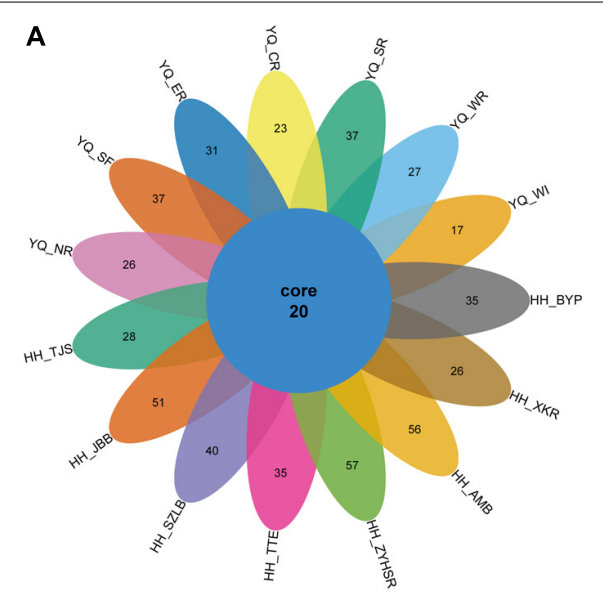

B
Haihe River

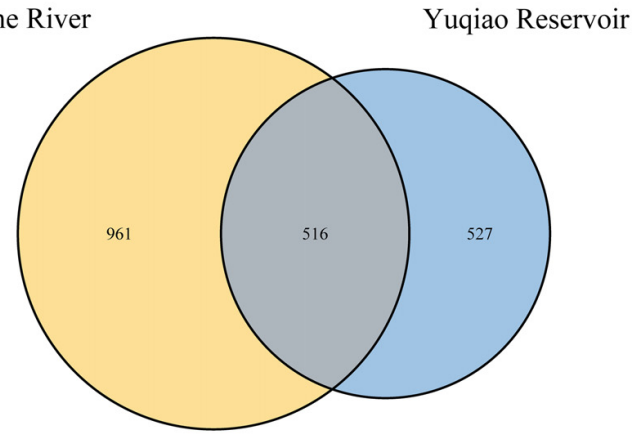

FIGURE 2 | Venn diagram of OTU numbers in 15 samples. Cluster at 97\% cut-off. (A) Individual samples. (B) Samples merged into groups.

TABLE 2 | Summary of papers related to Microcystis ITS genotypes detecting methods.

\begin{tabular}{lcccccc}
\hline Water body & Country & Method & Sequences & Dissimilarity & Genotypes & Reference \\
\hline Grangent Reservoir & France & Library cloning & 784 & One mutation & 306 & Briand et al., 2009 \\
Loire River & France & Library cloning & 950 & One mutation & 375 & Sabart et al., 2009 \\
Qinhuai River & China & Library cloning & 546 & One mutation & 230 & Xu et al., 2011 \\
Xing Pond & China & Library cloning & 563 & One mutation & 320 & Zhu et al., 2012 \\
Taihu-Chaohu & China & Library cloning & 84 & One mutation & 15 & Cai et al., 2012 \\
Erhai Lake & China & Library cloning & 800 & One mutation & 473 & Song et al., 2015 \\
Haihe-Yuqiao & China & High-throughput sequencing & $\mathbf{6 2 9 3 4 1}$ & $\mathbf{3 \%}$ of sequences & $\mathbf{2 0 0 5}$ & This study \\
\hline
\end{tabular}

\section{Multidimensional Analyses and Statistics}

All statistical analyses were performed in the $\mathrm{R}$ environment using VEGAN (Dixon, 2017) and grid (Wegener et al., 2009) packages. Results were presented as mean values \pm standard deviation. Alpha diversity was measured by (Quantitative Insights Into Microbial Ecology) QIIME procedure (Caporaso et al., 2010). OTU network was analyzed with QIIME and plotted using the R package Igraph (Csardi and Nepusz, 2006).
We selected the OTUs with $>1 \%$ abundance for the network analysis. Weighted Unifrac and Bray-Curtis Matrix were calculated with vegan package and plotted with pheatmap package. The correlation between environmental factors with OTUs was measured with Pearson's relative indices (data shown in Supplementary Table S3). Detrended correspondence analysis (DCA) was performed to determine the appropriate type of model for direct gradient analysis (Jongman et al., 1995). 
A shannon rarefraction plot

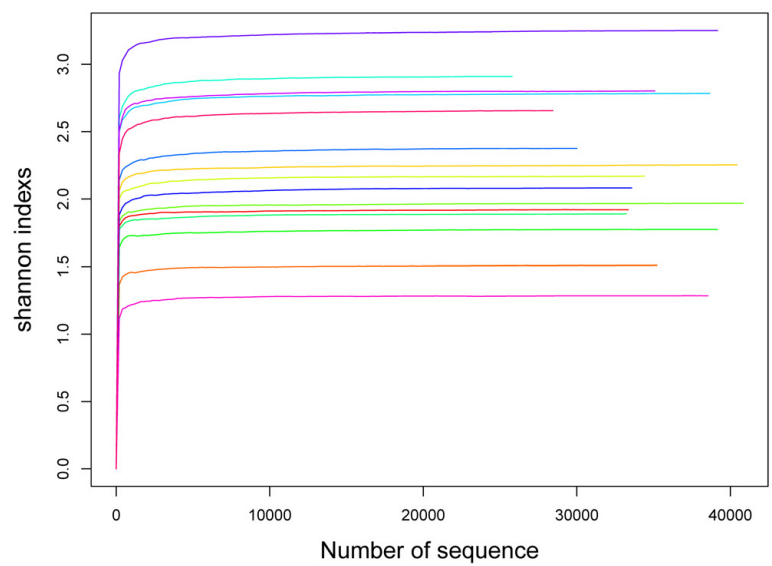

B

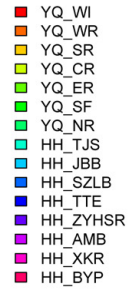

FIGURE 3 | Plots of alpha diversity analyses of sequences. (A) Shannon rare fraction curves. (B) Box plot of Simpson diversity.
Simpson diversity Plot

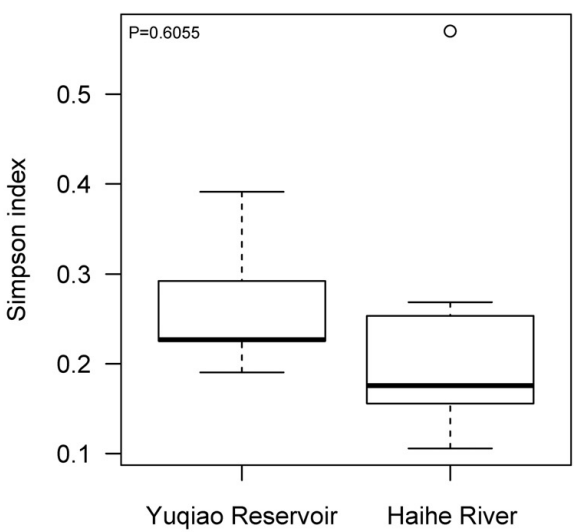

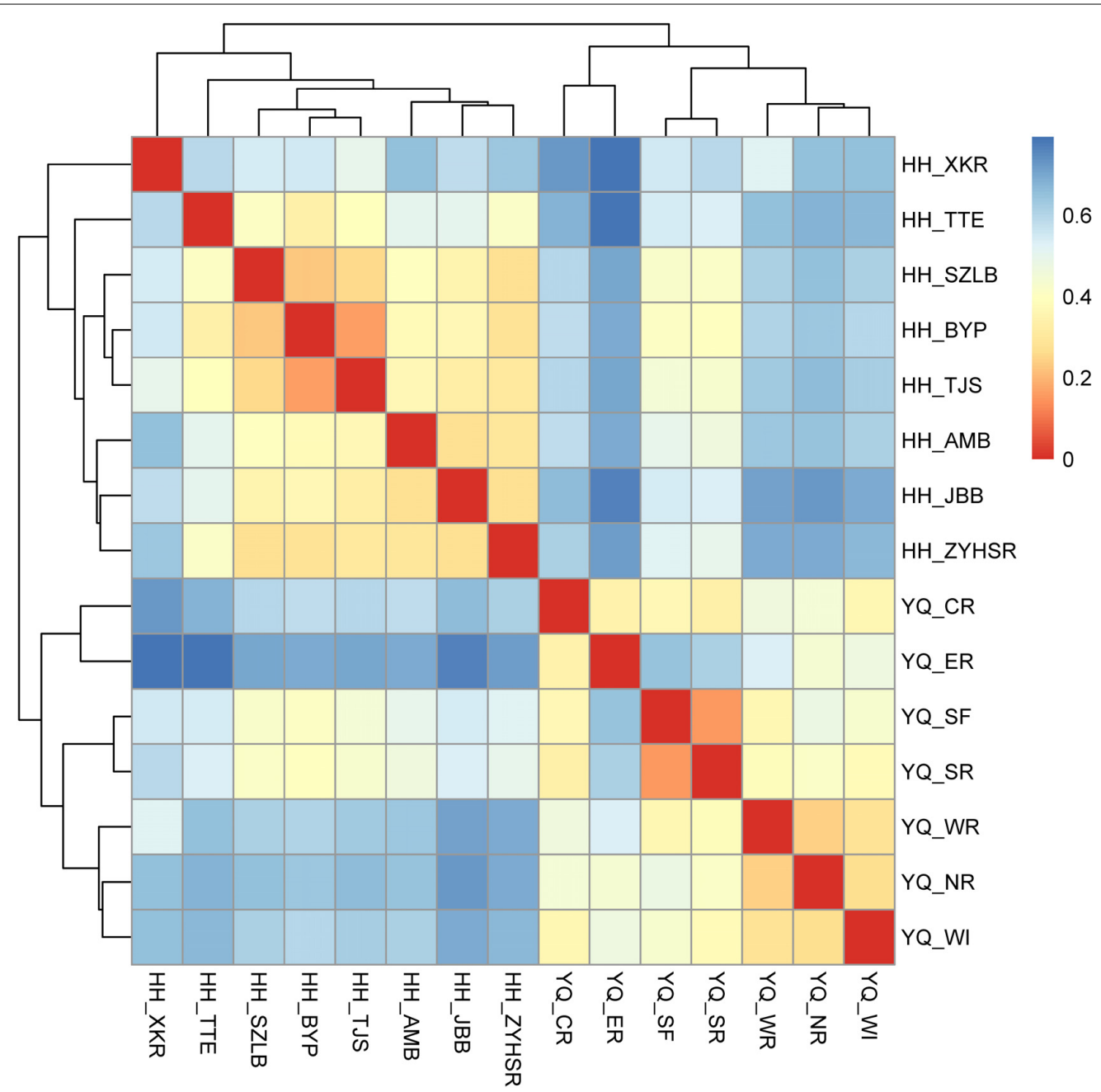

FIGURE 4 | Heatmap of weighted Unifrac distance. The color of square shows the distance between each two samples. The range of blue to red corresponds to far to close distance. 


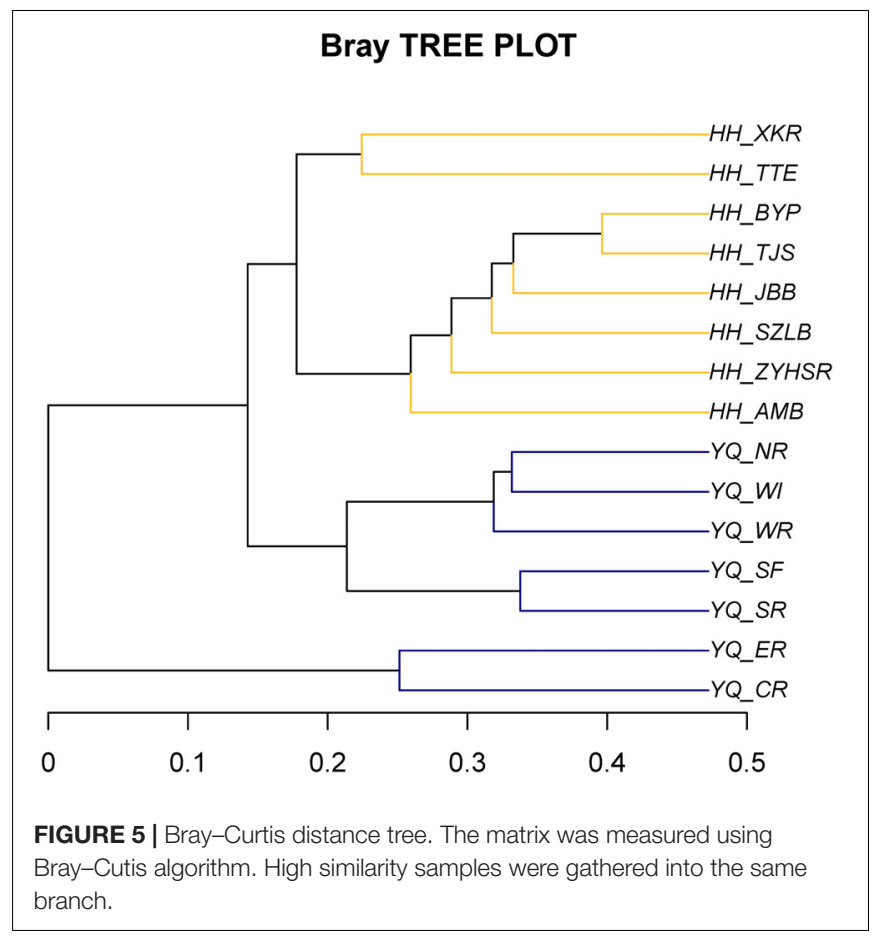

The environmental factors were transformed with $\log 10$ (OTU\#+1), and OTU abundances were transformed with Hellinger transformation (Legendre and Gallagher, 2001). DCA results show that a Unimodal model would be suitable for further analyses. Finally, canonical correlation analysis (CCA) was performed with Vegan package.

\section{Data Available}

The ITS amplicon sequences have been deposited as dataset SRP127241 in the sequence read archive (SRA) of the National Center for Biotechnology Information (NCBI).

\section{RESULTS}

\section{Sequencing Results and Alpha Diversity Analysis}

After quality control, we evaluated the distribution of all sequences, as shown in Supplementary Figure S1. All of the sequences obtained were $\sim 360$ bp-long. The singletons were removed for further analyses. A total of 629,341 reads were obtained. OTU numbers for each sample are listed in Table 1. HH_ZYHSR exhibited the highest OTU number of 664, whereas YQ_WI possessed the lowest OTU number of 245. The average OTU number in Haihe River was $497.6 \pm 123.3$, which was significantly higher than that in Yuqiao Reservoir (325.9 \pm 59.2). The Venn diagram in Figure 2A illustrated the common and unique OTUs in each sample. Among all samples, 20 OTUs commonly existed. HH_ZYHSR and HH_WI demonstrated the highest and lowest number of unique OTUs of 57 and 17, respectively. In two water areas, 516 common OTUs existed, and Haihe River contained double the number of unique OTUs as that of Yuqiao Reservoir (Figure 2B). From both views of sequencing depth and genotype numbers, HTS-based detection displayed an evidently higher efficiency than traditional library cloning method (Table 2). Shannon rarefaction plots indicated that most samples from the Haihe River showed higher genetic diversity than those from the Yuqiao Reservoir (Figure 3A). Simpson diversity plot also showed higher diversity in the Haihe River than that in the Yuqiao Reservoir (Figure 3B).

\section{Beta Diversity Analysis}

Weighted Unifrac distance heatmap showed that samples from the two water areas could be easily separated into two groups (Figure 4). Internal distance was close between Haihe River and Yuqiao Reservoir, with the two samples YQ_SF and YQ_SR as more closely related to the samples in Haihe River. The closest crossed group distance was found between YQ_SR and HH_BYP. Bray-Curtis-based distance tree also demonstrated clearly separated matrices between two water areas (Figure 5). HH_XKR and HH_TTE gathered into one group, whereas HH_BYP, HH_TJS, HH_JBB, HH_SZLB, HH_ZYHSR, and $\mathrm{HH}$ _AMB gathered into another. For the samples in Yuqiao reservoir, YQ_NR, YQ_WI, and YQ_WR were in one group, and YQ_SF and YQ_SR clustered together. YQ_ER and YQ_CR were distant from other samples. Clustered OTUs were measured by network analysis. Figure 6 illustrates that Otu0 was the most abundant in two water areas. This OTU network displayed a clear separation between Yuqiao Reservoir and Haihe River.

\section{Phylogenetic Analysis}

To further understand the genetic characters of ITS region, we compared the genotypes obtained in this study with those in previous studies. We constructed a neighbor-joining tree using representative sequences from top 20 abundant OTUs and the 100 previous sequences downloaded from NCBI.

The tree displayed no significant geographic distribution pattern (Figure 7). The dominant genotype Otu0 closely clustered with JN210304.1, which was detected in Xinghu pond, Wuhan, China (Zhu et al., 2012). Otu279, Otu33, Otu1, and Otu5 in this study were clustered together in a group, whereas Otu3, Otu293, Otu12, and OTu14 were clustered in another, indicating that these genotypes may possess regional characters that are unique from those obtained in previous studies. Interestingly, the results from phylogenetic and network analyses showed that most OTUs from Group 1 (Otu279, Otu1, and Otu5) dominated in Yuqiao reservoir but were rare in Haihe River. Conversely, Group 2 OTUs (Otu3, Otu293, Otu12, and Otu14) were mostly found in Haihe River but were rare in Yuqiao reservoir.

\section{Relationship Between OTU Abundance and Environmental Factors}

Environmental parameters revealed that Yuqiao Reservoir had a significantly higher eutrophication level than Haihe River, as shown in the result of Huo et al. (2018). (TSI Value in Yuqiao Reservoir: $64.94 \pm 2.46$ and in Haihe River: $50.02 \pm 10.78)$ Pearson correlation coefficient was calculated to evaluate the relationship between OTU abundance and 


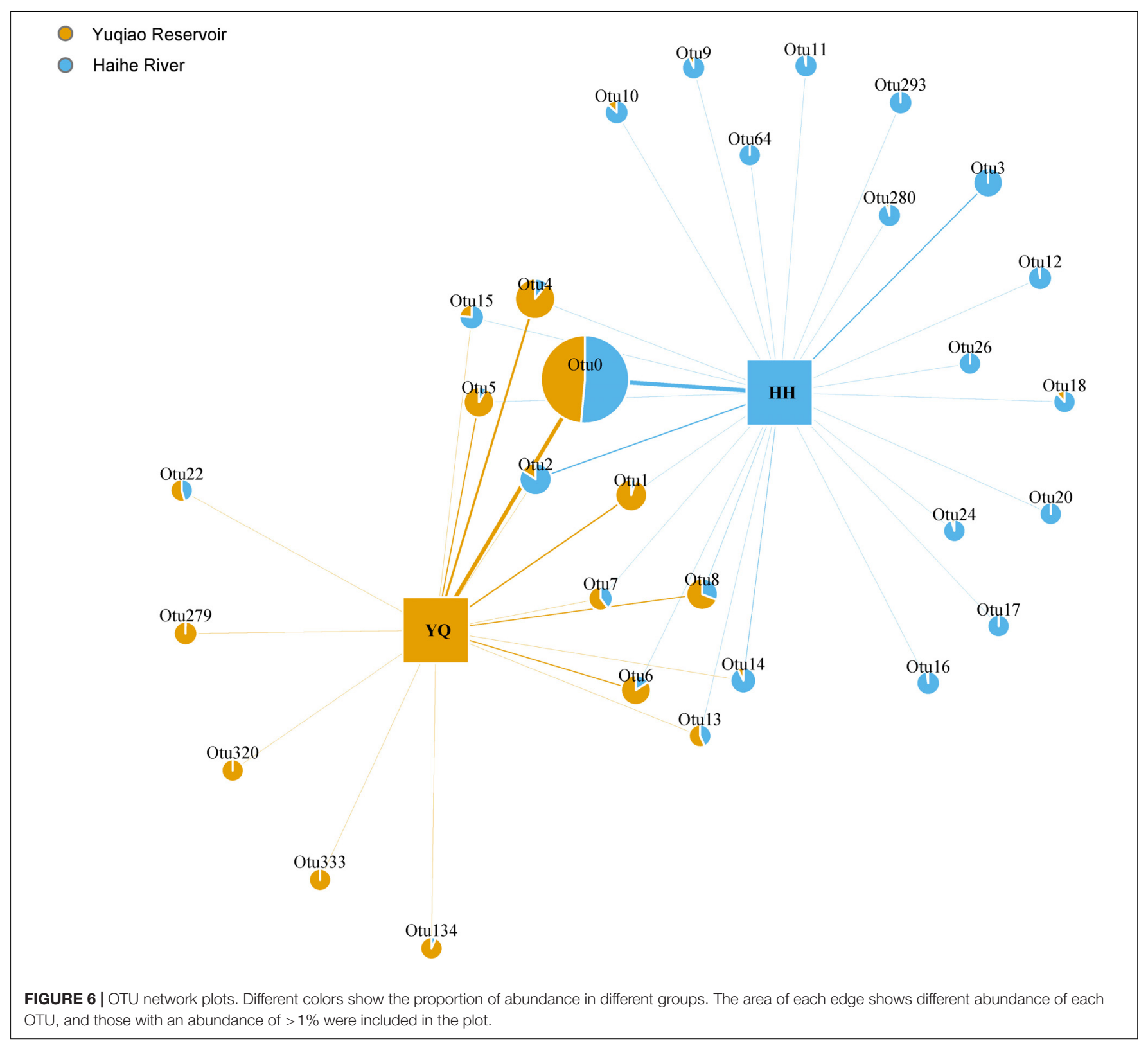

environmental factors (Results in Supplementary Table S3), and CCA was subsequently conducted to show all their correlations. The CCA ordination showed that OTU abundance was strongly affected by environmental factors (Figure 8). In general, samples from Haihe River clustered together on CCA plot and could be differentiated from samples in Yuqiao Reservoir. They were positively related to DO, SAL, and different forms of dissolved nitrogen. The samples in Yuqiao Reservoir were more discrete and most samples were positively related to $\mathrm{TN}, \mathrm{TP}$, and $\mathrm{WT}$. The specific OTU genotypes preferred various environmental conditions. The dominant OTU genotype Otu0 was positively and significantly affected by TN and TP $(P<0.05)$. WT, pH, and $\mathrm{NO}_{2}^{-}$also contributed to the distribution of Otu0, though not significantly $(P>0.05)$.

\section{DISCUSSION}

Using molecular biological approach to investigate the spatiotemporal dynamics of Microcystis populations has become an important tool for understanding Microcystis population genetics and the spatial development of a bloom in a water system (Kardinaal et al., 2007; Briand et al., 2009; Bozarth et al., 2010). Such molecular approaches have largely advanced from denaturing gradient gel electrophoresis and clone sequencing to HTS by next-generation sequencing. The present study is, to the best of our knowledge, the first application of HTS in examining Microcystis intra-species diversity on the ITS region. Previous works in detecting Microcystis genotype with library cloning could only provide dozens to thousands of sequences (Table 2). By defining 


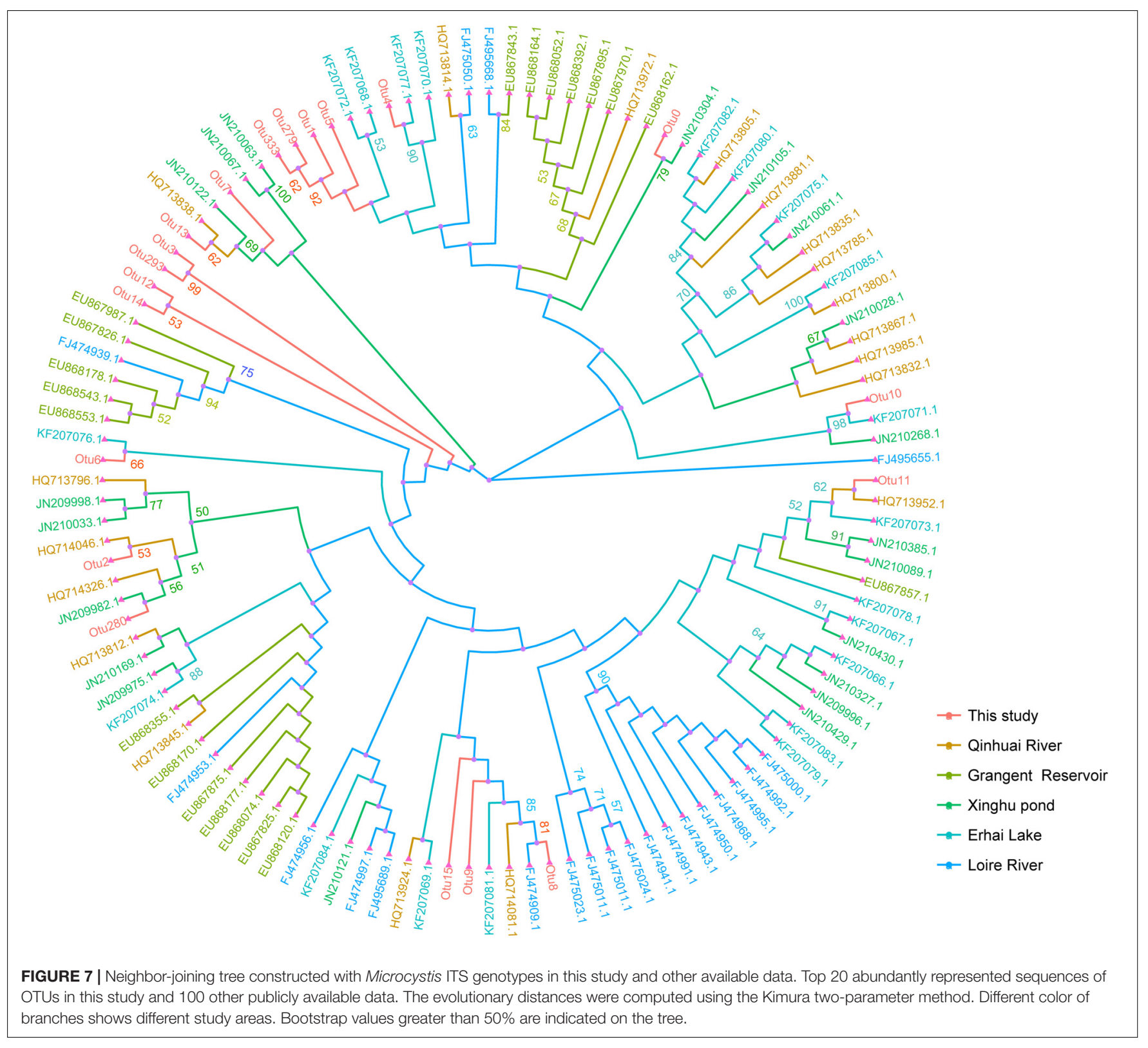

one base pair divergence as a new genotype, these previous studies using the cloning-based method could identify up to hundreds of genotypes in a water body, such as 618 genotypes from 2337 ITS sequences in Lake Taihu-the maximum number of ITS genotypes so far (Liu et al., 2016). The HTS result of the present study obtained 629,341 sequences in Yuqiao-Haihe water system. Although $97 \%$ cut-off was set as the OTU threshold, the thousands of OTUs identified in this study after removing singletons indicated that the Microcystis populations were extremely diverse at the ITS region. Therefore, assigning 97\% cut-off in ITS similarity as an OTU provides a highly reasonable definition to reveal divergence among each OTU and sample site. We verified that HTS brings much bigger sampling size than traditional library cloning method.
The comparison in the ITS genetic diversity of Microcystis blooms between the two water areas showed that Haihe River is more diverse than Yuqiao Reservoir. The environmental parameters detected already showed that Yuqiao was more eutrophicated than Haihe River. Thus, the genetic diversity of the ITS genotypes was negatively correlated with eutrophication level, which was very similar to the result obtained in Erhai Lake by Song et al. (2015). Yuqiao Reservoir is a semi-closed reservoir, and water from Yuqiao Reservoir flows into Haihe River as partial water resource. The different water resource flow-in may lead to an increased Microcystis intra-species diversity. Briand et al. (2009) reported that the variation in the dominant genotypes was transformational during a bloom and tracing the dominant species could also provide a way to find the seed source triggering the blooms (Xu et al., 2011). 


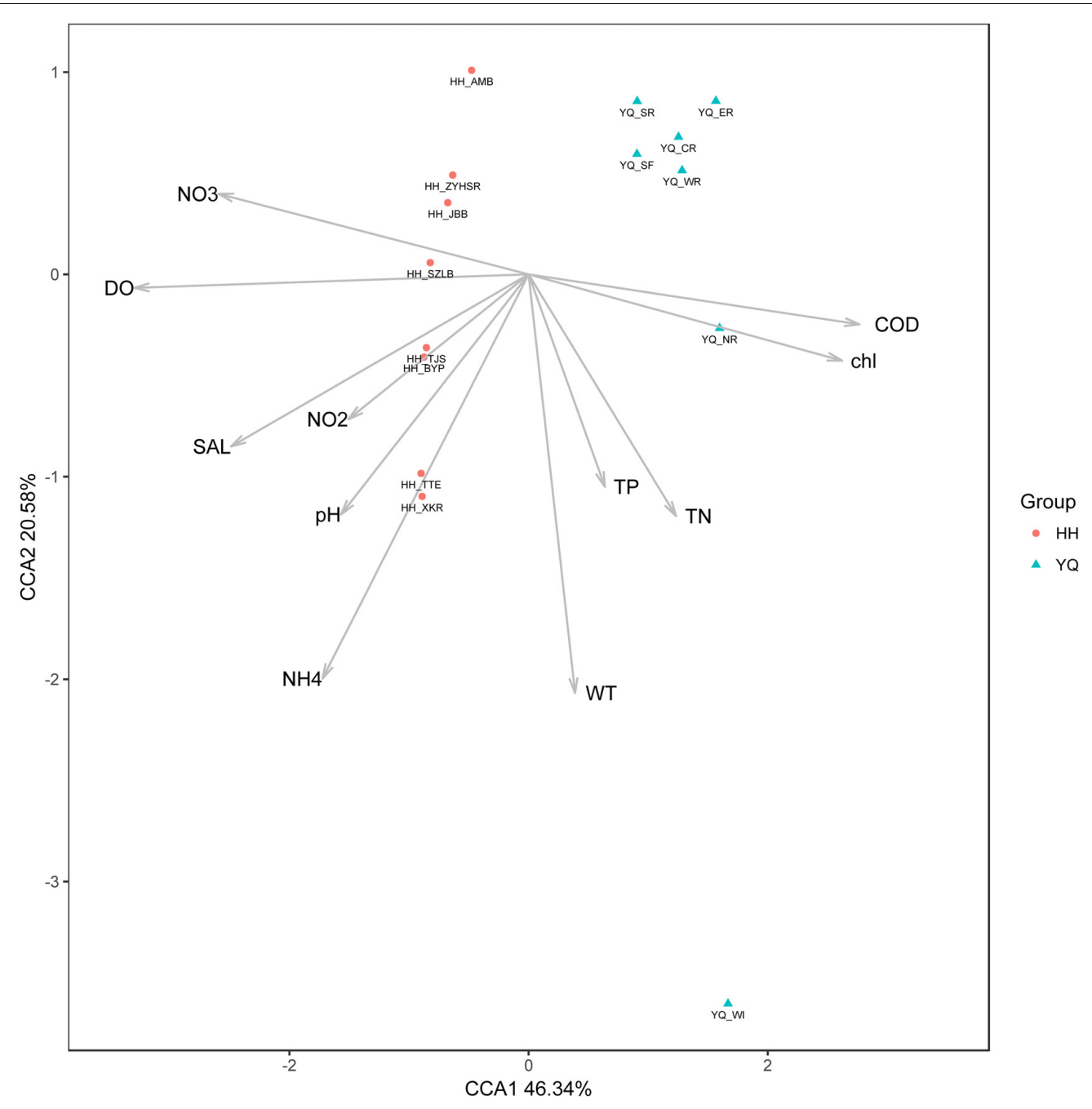

FIGURE 8 | Canonical correspondence analysis showing the relationships between environmental factors and the relative abundance of OTUs.

Through beta diversity analyses, a clearly separated relationship between two water areas was displayed. Most sites demonstrated different abundant characters between two water areas, but the samples from two sites in Yuqiao (YQ_SF and YQ_SR) were revealed to be closely related to those in Haihe River, indicating that Microcystis populations in these two sampling sites may contribute and adapt to the environmental conditions in Haihe River. Thus, these genotypes were maintained during water exchange in the two water areas. To better understand the factors driving the differentiation of genotypes, we investigated the relationship between environmental factors with different samples and genotypes. As shown in the results, the only dominant genotype Otu0 was affected by TN and TP (Figure 8), implying that TN and TP play an important role in the formation of dominant genotypes. The proportion of Otu0 abundance was almost 1:1 between two water areas, and the Otu0 point on CCA plot was almost located at the perpendicular bisector of the samples from the two water areas, implying that the dominance of Otu0 was attributed to its great adaptive capacity in both waters.
Phylogenetic analyses indicated that no significant spatial distribution was found in the pattern of dominant genotypes, which was similar to the result in Erhai Lake, Yunnan, China (Song et al., 2015). Interestingly, several dominant OTUs were clustered together in the phylogenetic tree (Group I and Group II), and these OTUs were mostly found in Yuqiao Reservoir but rare in Haihe River. Such clustering pattern indicated that these Microcystis genotypes exhibit their own evolutionary adaption by developing a group of mutants to satisfy the variation of habitats. But this kind of adaption could be effective only in a small region. In this study, these genotypes could not be adapted to environment in Haihe River. Thus, it was difficult to develop the Microcystis genotypes in Yuqiao Reservoir into Haihe River along the water flow. High genomic plasticity was reported for Microcystis (Kaneko et al., 2007; Frangeul and Al, 2008), and Microcystis species were shown to possess flexible genome evolution to integrate genes from other individuals (Zhang et al., 2016), which may explain why such similar genotypes were found at a small scale. 
The HTS-based approach used in this study demonstrated a high diversity of Microcystis genotypes coexisting in two water areas in Tianjin. Compared with traditional library cloning method, HTS provides more information for the comprehensive detection of environmental samples. With the development of HTS, additional advantages will be demonstrated. Future studies could monitor different proportions of each genotype during a bloom. The convenient and advanced method will provide the possibility to study diversity in Microcystis in larger scales.

\section{CONCLUSION}

Using the newly generated primer for detecting Microcystis ITS region by HTS, we examined Microcystis intra-species diversity along a Yuqiao-Haihe water system in Tianjin, China. The results demonstrated an extremely higher diversity of Microcystis in studied waters compared with that in previous studies using the traditional cloning sequencing. The ITS-based genetic diversity of Microcystis populations was negatively related to eutrophication levels, and the dominant genotype in two water areas was mostly affected by TN and TP. Finally, phylogenetic analyses revealed a possible flexible genotype evolution at a small scale at YuqiaoHaihe water system.

\section{AUTHOR CONTRIBUTIONS}

$\mathrm{ZQ}$ and RL designed the study. $\mathrm{DH}, \mathrm{YC}, \mathrm{TZ}, \mathrm{XL}$, and $\mathrm{XZ}$ performed the sampling and data analysis. $\mathrm{DH}$ wrote the manuscript. GY revised the manuscript and performed the primer checking experiment.

\section{REFERENCES}

Berry, M. A., White, J. D., Davis, T. W., Jain, S., Johengen, T. H., Dick, G. J., et al. (2017). Are oligotypes meaningful ecological and phylogenetic units? A case study of Microcystis in freshwater lakes. Front. Microbiol. 8:365. doi: 10.3389/fmicb.2017.00365

Bertos-Fortis, M., Farnelid, H. M., Lindh, M. V., Casini, M., Andersson, A., Pinhassi, J., et al. (2016). Unscrambling cyanobacteria community dynamics related to environmental factors. Front. Microbiol. 7:625. doi: 10.3389/fmicb. 2016.00625

Bonilla, S., Aubriot, L., Soares, M. C. S., González-Piana, M., Fabre, A., Huszar, V. L., et al. (2012). What drives the distribution of the bloomforming cyanobacteria Planktothrix agardhii and Cylindrospermopsis raciborskii. FEMS Microbiol. Ecol. 79, 594-607. doi: 10.1111/j.1574-6941.2011. 01242.x

Bozarth, C. S., Schwartz, A. D., Shepardson, J. W., Colwell, F. S., and Dreher, T. W. (2010). Population turnover in a Microcystis bloom results in predominantly nontoxigenic variants late in the season. Appl. Environ. Microbiol. 76, 5207-5213. doi: 10.1128/AEM.00001-10

Briand, E., Escoffier, N., Straub, C., Sabart, M., Quiblier, C., and Humbert, J. F. (2009). Spatiotemporal changes in the genetic diversity of a bloomforming Microcystis aeruginosa (cyanobacteria) population. ISME J. 3, 419-429. doi: 10.1038/ismej.2008.121

Cai, Y., Kong, F., Shi, L., and Yu, Y. (2012). Spatial heterogeneity of cyanobacterial communities and genetic variation of Microcystis populations within large, shallow eutrophic lakes (Lake Taihu and Lake Chaohu. China). J. Environ. Sci. 24, 1832-1842. doi: 10.1016/S1001-0742(11)61007-3

\section{FUNDING}

This study was supported by the National Science Foundation of China (Grant No. NSFC51779247) and International Science and Technology Cooperation Program of China (Grant No. 2013DFA71340). And we would like to thank for the support from Tianjin Agricultural University Key Laboratory of Aqua-ecology platform project.

\section{ACKNOWLEDGMENTS}

We would like to thank Yiming $\mathrm{Li}$ and Qi Cao at Tianjin Agricultural University for their work on sample collection. We also appreciate the advice in manuscript revision from Dr. Hua Li in Institute of Hydrobiology, Chinese Academy of Sciences.

\section{SUPPLEMENTARY MATERIAL}

The Supplementary Material for this article can be found online at: https://www.frontiersin.org/articles/10.3389/fmicb. 2018.00971/full\#supplementary-material

FIGURE S1 | Length distribution of reads.

TABLE S1 | Strains used for examination of Microcysits-specific ITS primers.

TABLE S2 | Online BLAST based primer checking results (OTUs with abundance $>10$ is involved).

TABLE S3 | Correlations between OTU abundance and environmental factors.

Caporaso, J. G., Kuczynski, J., Stombaugh, J., Bittinger, K., Bushman, F. D., Costello, E. K., et al. (2010). QIIME allows analysis of high-throughput community sequencing data. Nat. Methods 7, 335-336. doi: 10.1038/nmeth. f.303

Chen, C., Zhang, Z. C., Ding, A. Z., Wu, J. Y., Xiao, J. F., and Sun, Y. J. (2011). "BarCoded pyrosequencing reveals the bacterial community during Microcystis water bloom in guanting reservoir," in Proceedings of the Second Sree Conference on Chemical Engineering (Cce 2011), Beijing, 18.

Chislock, M. F., Doster, E., Zitomer, R. A., and Wilson, A. E. (2013). Eutrophication: causes, consequences, and controls in aquatic ecosystems. Nat. Educ. Knowl. 4:10.

Codd, G. A., Morrison, L. F., and Metcalf, J. S. (2005). Cyanobacterial toxins: risk management for health protection. Toxicol. Appl. Pharmacol. 203, 264-272. doi: 10.1016/j.taap.2004.02.016

Cristiana, M., Vitor, V., and Agostinho, A. (2013). Phylogeny and biogeography of cyanobacteria and their produced toxins. Mar. Drugs 11, 4350-4369. doi: $10.3390 / \mathrm{md} 11114350$

Csardi, G., and Nepusz, T. (2006). The Igraph software package for complex network research. Interj. Complex Syst. 1695, 1-9.

Danillo, O., Alvarenga, Marli, F., Fiore Alessandro, M., and Varani. (2017). A metagenomic approach to cyanobacterial genomics. Front. Microbiol. 8:809. doi: $10.3389 /$ fmicb.2017.00809

Dixon, P. (2017). VEGAN, A Package of R functions for community ecology. J. Veg. Sci. 14, 927-930. doi: 10.1111/j.1654-1103.2003.tb02228.x

Edgar, R. C. (2013). UPARSE: highly accurate OTU sequences from microbial amplicon reads. Nat. Methods 10, 996-998. doi: 10.1038/nmeth. 2604 
Edgar, R. C., Haas, B. J., Clemente, J. C., Christopher, Q., and Rob, K. (2011). UCHIME improves sensitivity and speed of chimera detection. Bioinformatics 27, 2194-2200. doi: 10.1093/bioinformatics/ btr381

Frangeul, L., and Al, E. (2008). Highly plastic genome of Microcystis aeruginosa PCC 7806, a ubiquitous toxic freshwater cyanobacterium. BMC Genomics 9:274. doi: 10.1186/1471-2164-9-274

Hall, T. A. (1999). BioEdit: a user-friendly biological sequence alignment editor and analysis program for Windows 95/98/NT. Nucleic Acids Symp. 41, 95-98.

Hess, W. R. (2011). Cyanobacterial genomics for ecology and biotechnology. Curr. Opin. Microbiol. 14, 608-614. doi: 10.1016/j.mib.2011.07.024

Huo, D., Chen, Y. X., Liu, P., Li, Y. M., Qiao, Z. Y., and Li, R. H. (2018). Molecular detection of microbial communities associated with Microcystis vs Synechococcus dominated waters in Tianjin, China. J. Oceanol. Limnol. (in press). doi: 10.1007/s00343-018-7182-x

Iteman, I., Rippka, R., Tandeau, D. M. N., and Herdman, M. (2000). Comparison of conserved structural and regulatory domains within divergent $16 \mathrm{~S}$ rRNA-23S rRNA spacer sequences of cyanobacteria. Microbiology 146(Pt 6), 1275-1286. doi: 10.1099/00221287-146-6-1275

Jin, X. C., and Tu, Q. Y. (1990). The Standard Methods for Observation and Analysis in Lake Eutrophication. Chinese Environmental. Beijing: Science Press, 240.

Jongman, R. H., Braak, C. J. F. T., and Tongeren, O. F. R. V. (1995). Data Analysis in Community and Landscape Ecology. Cambridge: Cambridge University Press, 10. doi: 10.1017/СBO9780511525575

Kaneko, T., Nakajima, N., Okamoto, S., Suzuki, I., Tanabe, Y., Tamaoki, M., et al. (2007). Complete genomic structure of the bloom-forming toxic cyanobacterium Microcystis aeruginosa NIES-843. DNA Res. 14, 247-256. doi: 10.1093/dnares/dsm026

Kardinaal, W. E. A., Tonk, L., Janse, I., Hol, S., Slot, P., Huisman, J., et al. (2007). Competition for light between toxic and nontoxic strains of the harmful cyanobacterium Microcystis. Appl. Environ. Microbiol. 73, 2939-2946. doi: 10.1128/AEM.02892-06

Kataoka, T., Homma, T., Nakano, S. I., Hodoki, Y., Ohbayashi, K., and Kondo, R. (2013). PCR primers for selective detection of intra-species variations in the bloom-forming cyanobacterium, Microcystis. Harmful Algae 23, 46-54. doi: 10.1016/j.hal.2012.12.003

Klanchui, A., Cheevadhanarak, S., Prommeenate, P., and Meechai, A. (2017). Exploring components of the $\mathrm{CO} 2$-Concentrating mechanism in alkaliphilic cyanobacteria through genome-based analysis. Comput. Struct. Biotechnol. J. 15, 340-350. doi: 10.1016/j.csbj.2017.05.001

Kumar, S., Nei, M., Dudley, J., and Tamura, K. (2008). MEGA: a biologistcentric software for evolutionary analysis of DNA and protein sequences. Brief. Bioinform. 9, 299-306. doi: 10.1093/bib/bbn017

Legendre, P., and Gallagher, E. D. (2001). Ecologically meaningful transformations for ordination of species data. Oecologia 129, 271-280. doi: 10.1007/ s004420100716

Lemaire, V., Brusciotti, S., Van Gremberghe, I., Vyverman, W., Vanoverbeke, J., and De Meester, L. (2012). Genotype $\times$ genotype interactions between the toxic cyanobacterium Microcystis and its grazer, the waterflea Daphnia. Evol. Appl. 5, 168-182. doi: 10.1111/j.1752-4571.2011.00225.x

Li, D., Kong, F., Shi, X., Ye, L., Yu, Y., and Yang, Z. (2012). Quantification of microcystin-producing and non-microcystin producing Microcystis populations during the 2009 and 2010 blooms in Lake Taihu using quantitative real-time PCR. J. Environ. Sci. 24, 284-290. doi: 10.1016/S1001-0742(11) 60745-6

Liu, Y., Xu, Y., Wang, Z., Xiao, P., Yu, G., Wang, G., et al. (2016). Dominance and succession of Microcystis genotypes and morphotypes in Lake Taihu, a large and shallow freshwater lake in China. Environ. Pollut. 219, 399-408. doi: 10.1016/j.envpol.2016.05.021

Lobo, I. (2012). Basic local alignment search tool (BLAST). J. Mol. Biol. 215, $403-410$.

Mackey, K. R. M., Huntercevera, K., Britten, G. L., Murphy, L. G., Sogin, M. L., and Huber, J. A. (2017). Seasonal Succession and spatial patterns of Synechococcus microdiversity in a salt marsh estuary revealed through $16 \mathrm{~S}$ rRNA gene oligotyping. Front. Microbiol. 8:1496. doi: 10.3389/fmicb.2017. 01496

Martin, M. (2011). Cutadapt removes adapter sequences from high-throughput sequencing reads. EMBnet J. 17, 10-12. doi: 10.14806/ej.17.1.200
Moreira, C., Vasconcelos, V., and Antunes, A. (2016). Genetic characterization of Microcystis aeruginosa isolates from Portuguese freshwater systems. World J. Microbiol. Biotechnol. 32:118. doi: 10.1007/s11274-0162063-y

Nübel, U., Garciapichel, F., and Muyzer, G. (1997). PCR primers to amplify 16S rRNA genes from cyanobacteria. Appl. Environ. Microbiol. 63, 3327-3332.

O'neil, J. M., Davis, T. W., Burford, M. A., and Gobler, C. J. (2012). The rise of harmful cyanobacteria blooms: the potential roles of eutrophication and climate change. Harmful Algae 14, 313-334. doi: 10.1016/j.hal.2011.10.027

Otten, T. G., Crosswell, J. R., Mackey, S., and Dreher, T. W. (2015). Application of molecular tools for microbial source tracking and public health risk assessment of a Microcystis bloom traversing $300 \mathrm{~km}$ of the Klamath River. Harmful Algae 46, 71-81. doi: 10.1016/j.hal.2015.05.007

Otten, T. G., Paerl, H. W., Dreher, T. W., Kimmerer, W. J., and Parker, A. E. (2017). The molecular ecology of Microcystis sp. Blooms in the San Francisco Estuary Delta. Environ. Microbiol. 19, 3619-3637. doi: 10.1111/1462-2920.13860

Paerl, H. W., and Huisman, J. (2008). Climate - Blooms like it hot. Science 320, 57-58. doi: 10.1126/science.1155398

Paerl, H. W., Xu, H., Hall, N. S., Rossignol, K. L., Joyner, A. R., Zhu, G., et al. (2015). Nutrient limitation dynamics examined on a multi-annual scale in Lake Taihu, China: implications for controlling eutrophication and harmful algal blooms. J. Freshw. Ecol. 30, 5-24. doi: 10.1080/02705060.2014.994047

Parulekar, N. N., Kolekar, P., Jenkins, A., Kleiven, S., Utkilen, H., Johansen, A., et al. (2017). Characterization of bacterial community associated with phytoplankton bloom in a eutrophic lake in South Norway using 16S rRNA gene amplicon sequence analysis. PLoS One 12:e0173408. doi: 10.1371/journal.pone.0173408

Pobel, D., Godon, J.-J., Humbert, J.-F., and Robin, J. (2012). High-frequency monitoring of the genetic diversity and the potential toxicity of a Microcystis aeruginosa bloom in a French shallow lake. FEMS Microbiol. Ecol. 79, 132-141. doi: 10.1111/j.1574-6941.2011.01203.x

Ruegger, P. M., Clark, R. T., Weger, J. R., Braun, J., and Borneman, J. (2014). Improved resolution of bacteria by high throughput sequence analysis of the rRNA internal transcribed spacer. J. Microbiol. Methods 105, 82-87. doi: 10.1016/j.mimet.2014.07.001

Sabart, M., Pobel, D., Latour, D., Robin, J., Salençon, M. J., and Humbert, J. F. (2009). Spatiotemporal changes in the genetic diversity in French bloomforming populations of the toxic cyanobacterium, Microcystis aeruginosa. Environ. Microbiol. Rep. 1, 263-272. doi: 10.1111/j.1758-2229.2009.00042.x

Saitou, N., and Nei, M. (1987). The neighbor-joining method: a new method for reconstructing phylogenetic trees. Mol. Biol. Evol. 24, 189-204. doi: 10.1007/ BF02099966

Schmieder, R., and Edwards, R. (2011). Quality control and preprocessing of metagenomic datasets. Bioinformatics 27, 863-864. doi: 10.1093/ bioinformatics/btr026

Song, G., Jiang, Y., Yu, G., and Li, R. (2015). Genotypes of ITS region of rRNA in Microcystis (Cyanobacteria) populations in Erhai Lake (China) and their correlation with eutrophication level. J. Basic Microbiol. 55, 1203-1211. doi: $10.1002 /$ jobm. 201500080

Soo, R. M., Skennerton, C. T., Sekiguchi, Y., Imelfort, M., Paech, S. J., Dennis, P. G., et al. (2014). An expanded genomic representation of the phylum Cyanobacteria. Genome Biol. Evol. 6, 1031-1045. doi: 10.1093/gbe/evu073

Srivastava, A., Choi, G. G., Ahn, C. Y., Oh, H. M., Ravi, A. K., and Asthana, R. K. (2012). Dynamics of microcystin production and quantification of potentially toxigenic Microcystis sp. using real-time PCR. Water Res. 46, 817-827. doi: 10.1016/j.watres.2011.11.056

Stucken, K., Murillo, A. A., Soto-Liebe, K., Fuentes-Valdés, J. J., Méndez, M. A. and Vásquez, M. (2009). Toxicity phenotype does not correlate with phylogeny of Cylindrospermopsis raciborskii strains. Syst. Appl. Microbiol. 32, 37-48. doi: 10.1016/j.syapm.2008.10.002

Tan, W., Liu, Y., Wu, Z., Lin, S., Yu, G., Yu, B., et al. (2010). cpc BA-IGS as an effective marker to characterize Microcystis wesenbergii (Komárek) Komárek in Kondrateva (cyanobacteria). Harmful Algae 9, 607-612. doi: 10.1016/j.hal.2010. 04.011

Thompson, C. C., Chimetto, L., Edwards, R. A., Swings, J., Stackebrandt, E., and Thompson, F. L. (2013). Microbial genomic taxonomy. BMC Genomics 14:913. doi: 10.1186/1471-2164-14-913

Verspagen, J. M., Van De Waal, D. B., Finke, J. F., Visser, P. M., Van Donk, E., and Huisman, J. (2014). Rising CO2 levels will intensify phytoplankton blooms in 
eutrophic and hypertrophic lakes. PLoS One 9:e104325. doi: 10.1371/journal. pone.0104325

Visser, P. M., Verspagen, J. M. H., Sandrini, G., Stal, L. J., Matthijs, H. C. P., Davis, T. W., et al. (2016). How rising CO2 and global warming may stimulate harmful cyanobacterial blooms. Harmful Algae 54, 145-159. doi: 10.1016/j.hal.2015.12.006

Walter, J. M., Coutinho, F. H., Dutilh, B. E., Swings, J., Thompson, F. L., and Thompson, C. C. (2017). Ecogenomics and taxonomy of Cyanobacteria phylum. Front. Microbiol. 8:2132. doi: 10.3389/fmicb.2017. 02132

Wegener, D., Sengstag, T., Sfakianakis, S., Rüping, S., and Assi, A. (2009). GridR: an R-based tool for scientific data analysis in grid environments. Fut. Gen. Comput. Syst. 25, 481-488. doi: 10.1016/j.future.2008.09.004

Whitton, B. (2012). Ecology of Cyanobacteria II: Their Diversity in Space and Time. Berlin: Springer. doi: 10.1007/978-94-007-3855-3

Wittmann, C., and Liao, J. C. (2016). 15. Cyanobacteria as a Host Organism. Weinheim: Wiley-VCH Verlag GmbH \& Co. KGaA.

Xu, Y., Wang, G., Yang, W., and Li, R. (2010). Dynamics of the water bloomforming Microcystis and its relationship with physicochemical factors in Lake Xuanwu (China). Environ. Sci. Pollut. Res. 17, 1581-1590. doi: 10.1007/s11356010-0345-8

Xu, Y., Yang, F., Liu, Y., Wang, Z., Wang, J., Wang, G., et al. (2011). Genetic diversity of Microcystis populations in a bloom and its relationship to the environmental factors in Qinhuai River, China. Microbiol. Res. 167, 20-26. doi: 10.1016/j.micres.2011.02.005

Yoshida, M., Yoshida, T., Satomi, M., Takashima, Y., Hosoda, N., and Hiroishi, S. (2008). Intra-specific phenotypic and genotypic variation in toxic cyanobacterial Microcystis strains. J. Appl. Microbiol. 105, 407-415. doi: $10.1111 /$ j.1365-2672.2008.03754.x
Yu, G., Smith, D. K., Zhu, H., Guan, Y., and Lam, T. T. Y. (2017). ggtree: an $r$ package for visualization and annotation of phylogenetic trees with their covariates and other associated data. Methods Ecol. Evol. 8, 28-36. doi: 10.1111/ 2041-210X.12628

Zhang, J. J., Kobert, K., Flouri, T., and Stamatakis, A. (2014). PEAR: a fast and accurate Illumina Paired-End reAd mergeR. Bioinformatics 30, 614-620. doi: 10.1093/bioinformatics/btt593

Zhang, J. Y., Guan, R., Zhang, H. J., Li, H., Xiao, P., Yu, G. L., et al. (2016). Complete genome sequence and genomic characterization of Microcystis panniformis FACHB 1757 by third-generation sequencing. Stand. Genomic Sci. 11:11. doi: 10.1186/s40793-016-0130-5

Zhang, Q., Li, M., Liu, H., and Lei, L. (2009). Phylogenetic relationships of Microcystis based on rpoC1 gene sequences in China. Ecol. Environ. Sci. 18, 2039-2043. doi: 10.16258/j.cnki.1674-5906.2009.06.036

Zhu, M. L., Xu, Y., and Li, R. H. (2012). Genetic diversity of bloom-forming Microcystis (Cyanobacteria) populations in a hyper-eutrophic pond in central China. Curr. Microbiol. 65, 219-224. doi: 10.1007/s00284-012-0146-z

Conflict of Interest Statement: The authors declare that the research was conducted in the absence of any commercial or financial relationships that could be construed as a potential conflict of interest.

Copyright (c) 2018 Huo, Chen, Zheng, Liu, Zhang, Yu, Qiao and Li. This is an open-access article distributed under the terms of the Creative Commons Attribution License (CC BY). The use, distribution or reproduction in other forums is permitted, provided the original author(s) and the copyright owner are credited and that the original publication in this journal is cited, in accordance with accepted academic practice. No use, distribution or reproduction is permitted which does not comply with these terms. 